\title{
DERIVATION \\ OF A GENERAL LORENTZ TRANSFORMATION WITHOUT ROTATION
}

\author{
A. H. KLOTZ
}

(Received 20 February 1968; revised 29 April 1968)

\section{1}

The purpose of this note is to show that the standard form of a general Lorentz transformation without special rotation [1], [2], [3] can be derived from simple algebraic hypotheses.

Let Greek indices go from 1 to 4 and Latin indices from 1 to 3 . Summation convention over repeated indices in a product is used throughout and the velocity of light $c$ is taken to be unity.

A Lorentz transformation in a four dimensional space-time is defined by the relations

where

$$
x_{\mu}^{\prime}=a_{\mu \nu} x_{\nu}, \quad x_{\mu}=a_{\nu \mu} x_{\nu^{\prime}}^{\prime}
$$

$$
a_{\mu \lambda} a_{\mu \nu}=a_{\lambda \mu} a_{\nu \mu}=\delta_{\lambda \nu},
$$

$\delta_{\lambda \nu}$ being the Kronecker delta tensor.

\section{2}

Let

(2)

$$
\begin{aligned}
& u_{i}=\frac{x_{i}}{x_{4}} \quad \text { when } \quad x_{i}^{\prime}=0, \quad \text { and } \\
& u_{i}^{\prime}=\frac{x_{i}^{\prime}}{x_{4}^{\prime}} \quad \text { when } \quad x_{i}=\mathbf{0} .
\end{aligned}
$$

Then

$$
u_{i}^{\prime}=\frac{a_{i 4}}{a_{44}}, \quad u_{i}=\frac{a_{4 i}}{a_{44}} .
$$

A Lorentz transformation without spatial rotation is defined by the condition that

$$
u_{i}^{\prime}=-u_{i}, \quad \begin{aligned}
& \text { or } \\
& 141
\end{aligned} \quad a_{i 4}=-a_{4 i} .
$$


Let us write

$$
a_{i 4}=i \beta v_{i}, \quad a_{44}=\beta,
$$

where $i=\sqrt{ }-1$ and $v_{i}$ are all real in the usual interpretation of the coordinates $x_{\mu}$ in a Minkowski space. If $\lambda=v=4$ in (1), it follows that

$$
\beta^{2}=\left(1-v^{2}\right)^{-1}, \quad v^{2}=v_{i} v_{i} .
$$

Similarly

$$
a_{i j} a_{i k}+a_{4 j} a_{4 k}=\delta_{j k}=a_{i j} a_{i k}-\beta^{2} v_{j} v_{k}
$$

and

$$
a_{i 4} a_{i j}+a_{44} a_{4 j}=0
$$

Hence

$$
a_{i j} v_{i}=\beta v_{j}
$$

and

$$
a_{i j} a_{i k}-a_{i j} a_{l k} v_{i} v_{l}=\delta_{j k}=a_{i j} a_{l k} \rho_{i l}
$$

say, where

$$
p_{i l}=p_{l i}=\delta_{i l}-v_{i} v_{l} .
$$

Replacing the coefficients in (9) by their transposed (this is justified in view of (1)), multiplying both sides by $v_{j}$ and using the symmetry of $p_{i j}$,

Also, from (10),

$$
a_{j i} v_{j} p_{i l} a_{k l}=v_{k}=a_{k l} p_{i l} v_{i} .
$$

Hence

$$
p_{i l} v_{i}=v_{l}-v^{2} v_{l}=v_{l} \beta^{-2} \text {. }
$$

$$
\beta v_{k}=a_{k l} v_{l} .
$$

Comparison of (8) and (11) shows that the skewsymmetric part $a_{i j}$ of $a_{i j}$, satisfies the equations

$$
a_{i j} v_{j}=0
$$

Hence, $a_{i j}$ may be written in the form

$$
a_{i j}=\alpha\left\{\begin{array}{rrr}
0 & v_{3} & -v_{2} \\
-v_{3} & 0 & v_{1} \\
v_{2} & -v_{1} & 0
\end{array}\right\}=\alpha s_{i j} \text {, say, }
$$

where $\alpha$ is a parameter which may depend on $v_{i}$. Let us assume that the symmetric part $a_{\underline{i j}}$ of $a_{i j}$ is given by

$$
a_{\underline{i j}}=\delta_{i j}+q v_{i} v_{j}
$$


where $q$ may again depend on $v_{i}$. Hence, from (8),

$$
q=\frac{\beta-1}{v^{2}} \text {. }
$$

Thus, the most general Lorentz transformation of the type required is

By (1) we now have

$$
a_{i j}=\delta_{i j}+q v_{i} v_{j}+\alpha s_{i j} \text {. }
$$

$$
\alpha^{2} s_{i j} s_{i k}=0,
$$

and this cannot be satisfied by an arbitrary real vector

$$
\begin{aligned}
& \underline{v}=\left(v_{1}, v_{2}, v_{3}\right) \text { unless } \\
& \alpha=0 .
\end{aligned}
$$

Therefore the transformation is given by

$$
a_{i j}=\delta_{i j}+\frac{\beta-1}{v^{2}} v_{i} v_{j}, \quad a_{i 4}=-a_{4 i}=i \beta v_{i}, \quad a_{44}=\beta,
$$

as required (ref. 1).

Consider the transformation (18) followed by an infinitesimal Lorentz transformation without spacial rotation. The latter is defined by

$$
b_{i j}=\delta_{i j}, \quad b_{i 4}=-b_{4 i}=i u_{i}, \quad b_{44}=1 .
$$

The coefficients of the combined transformation are

$$
c_{\mu \nu}=b_{\mu \lambda} a_{\lambda \nu},
$$

so that

$$
\begin{aligned}
c_{4 j} & =b_{4 i} a_{i j}+b_{44} a_{4 j} \\
& =-i u_{i}\left(\delta_{i j}+\frac{v_{i}-v_{j}}{v^{2}}(\beta-1)\right)-i \beta v_{j} \\
& =-i \beta\left(u_{j}+v_{j}\right)+i \frac{\beta-1}{v^{2}}\left(v_{i} v_{i} u_{j}-u_{i} v_{i} v_{j}\right), \\
c_{j 4} & =i \beta\left(u_{j}+v_{j}\right),
\end{aligned}
$$

and

$$
c_{44}=\beta\left(1+u_{j} v_{j}\right) .
$$

Since the condition for the absence of spatial rotation is still 


$$
c_{j 4}=-c_{4 j},
$$

it follows that successive transformations of the above type induce a rotation of the space axes of the original coordinate system. This rotation is of the amount (from (21))

$$
\frac{\beta-1}{v^{2}} \frac{v_{i} v_{i} u_{j}-u_{i} v_{i} j_{j}}{1+u_{i} v_{j}}=\frac{\beta-1}{\beta v^{2}} \frac{\underline{v}_{\wedge}\left(\underline{u}_{\wedge} \underline{v}\right)}{1+\underline{u} \cdot \underline{v}} .
$$

For infinitesimal $\underline{u}=d \underline{u}$, this gives the precession operator

$$
-\frac{\beta-1}{\beta v^{2}} \underline{v}_{\wedge} d u
$$

or the well known Thomas precession

$$
-\frac{\underline{v} \wedge \underline{v}}{2}
$$

when $v$ is such smaller than the speed of light.

The author wishes to express his gratitude to Mr. R. Johnston of Liverpool University, England, to his colleague Mr. L. Karlov, for valuable discussions and to the Referee for a constructive criticism.

\section{Summary}

An elementary, purely algebraic derivation of the most general Lorentz transformations without spacial rotation and of the Thomas precession is given.

\section{References}

[1] C. Möller, The Theory of Relativity (Oxford, 1952).

[2] G. Stephenson and C. W. Kilmister, Special Relativity for Physicists (London, 1958).

[3] W. H. Furry, Am. J. Phys. 23 (8) (1955), 517-25.

Department of Applied Mathematics

University of Sydney 